\title{
Two-phase flow experiments with Coriolis Mass Flow Metering using complex signal processing
}

\author{
Ming Li ${ }^{\mathrm{a}}$, Manus Henry ${ }^{\mathrm{a}, \mathrm{b}, *}$, Feibiao Zhou ${ }^{\mathrm{a}}$, Michael Tombs ${ }^{\mathrm{a}}$ \\ ${ }^{\mathrm{a}}$ University of Oxford, UK \\ ${ }^{\mathrm{b}}$ South Ural State University, Russian Federation
}

\section{A R T I C L E I N F O}

\section{Keywords:}

Coriolis mass flow meter

Two-phase flow

Signal processing

Complex bandpass filtering

System-on-chip

\begin{abstract}
A B S T R A C T -
Two-phase (gas/liquid) flow is common in many industrial applications but its measurement remains challenging for Coriolis mass flow meters (CMFM), especially for high Gas Void Fraction (GVF). In this paper, we present experimental results applying previously developed complex signal processing techniques for tracking the rapidly changing sensor signals generated by two-phase flow. The techniques are implemented in a new System-on-Chip (SOC) prototype transmitter connected to a commercial Coriolis flow tube. Experiments have been carried out over a range of single phase and two-phase (water/air) flows. The signal tracking as well as mass flow rate and density measurement performance is compared with that of a laboratory version of a commercial Coriolis transmitter with two-phase flow capability. The results show that the complex bandpass algorithms, coupled with flowtube control algorithms, reduce the standard deviation of the mass flow measurement by a factor of 3 or more in $50 \%$ of the experiments undertaken. For the density measurement, the corresponding reduction in standard deviation is by a factor of 6 .
\end{abstract}

\section{Introduction}

THE Coriolis Mass Flow Meter (CMFM) measures the mass flow rate and density of a pure fluid (liquid or gas) to high accuracy. The CMFM uses the interaction between the flowing material and the flowtube to create a Coriolis acceleration on the flowing material and to sense the reaction on the flow tube [1,2]. An overview of the development of CMFM over the last 20 years is given in Ref. [3]. The meter is widely used in industrial applications in the oil and gas, food and beverage, chemical and pharmaceutical sectors. It also provides reference measurement data in experimental research domains, including combustion engines, heat exchangers, and thermal management [3-9]. Although CMFM has been called the "almost perfect" flow meter [10], two-phase (gas/liquid) conditions present challenges at two levels. The more widely recognized challenge is the large systematic errors, categorised here as fluid measurement errors, in the mass flow and density measurements induced by two phase conditions [11-22]; a range of correction techniques have been developed [23-29]. A yet more basic challenge is the rapid variations in the sinusoidal sensor signals induced by two-phase flow. These variations are a challenge to the signal processing algorithm which tracks frequency, amplitude and phase; errors in the tracking of these parameters are categorised here as signal tracking errors, and these affect the quality of flowtube oscillation control as well as feeding into the fluid measurement errors [30]. The main target of the research described here is this second challenge, i.e. developing signal processing techniques to reduce the signal tracking error in the frequency, phase, and amplitude estimations that occur under two phase flow conditions. This also indirectly addresses the first challenge, the systematic density and mass flow errors, by aiming to significantly reduce the instantaneous noise on these measurements, thereby facilitating improved corrections. Figs. 1 and 2 illustrate the impact of the two different error types on the mass flow rate and density during a series of experiments where the water mass flow rate is kept constant at $0.5 \mathrm{~kg} / \mathrm{s}$, while the GVF is increased from $1 \%$ up to $85 \%$. The results were obtained using the 'Oxbox' [30], a laboratory version of a commercial transmitter, which has been used for a number of multiphase flow applications [24,31]. Measurement updates are provided at approximately $200 \mathrm{~Hz}$, and the mean and standard deviation of the mass flow and density values (recorded in $120 \mathrm{~s}$ experiments with nominally steady reference conditions) are plotted as they vary with GVF. Our assumption here (as verified by later results) is that much of the mass flow and density measurement variation (as quantified by the standard deviation) arises not from genuine instantaneous changes in the gas/liquid mixture inside the flowtube, but rather from signal processing errors when tracking the rapidly changing sensor signals. These signal tracking errors then feed into the fluid

\footnotetext{
* Corresponding author. Department of Engineering Science, Parks Road, Oxford, OX1 3PJ, UK.

E-mail address: manus.henry@eng.ox.ac.uk (M. Henry).
} 


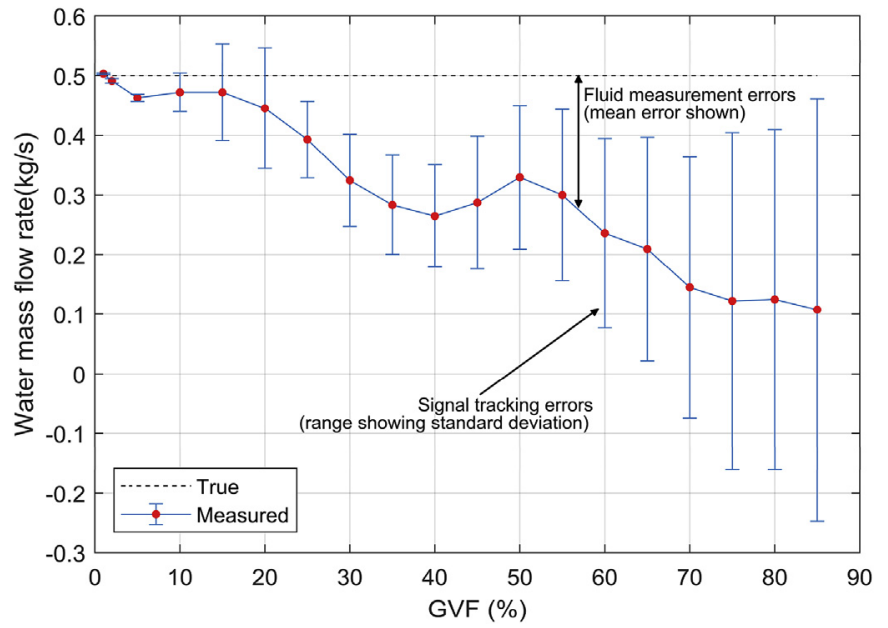

Fig. 1. Errors in two-phase mass flow rate measurement.

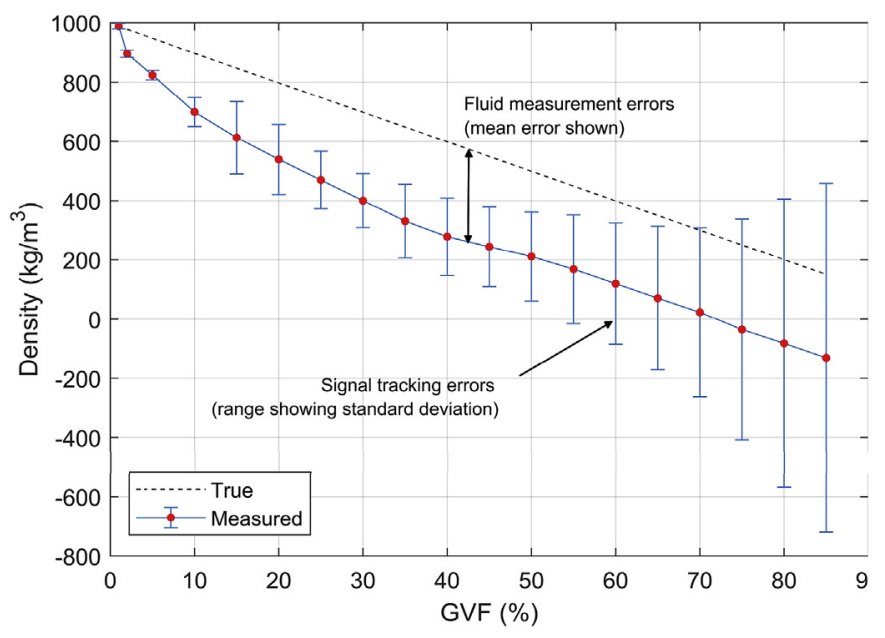

Fig. 2. Errors in two-phase density measurement.

measurement calculations. The large variations in fluid measurements have a detrimental influence on both the development of error corrections models (typically undertaken off-line), and the application of real-time correction based on such models. Hence reducing signal tracking errors makes a valuable contribution towards improving the final corrected two-phase flow measurement results.

In Ref. [32,33], a family of complex bandpass filtering and tracking algorithms was introduced, offering both good noise suppression and a fast dynamic performance, which is desirable in Coriolis applications beyond multiphase flow [34-36]. In Ref. [33], simulation studies demonstrated that these techniques out-performs a number of commonlyused CMFM signal processing algorithms, in terms for both noise reduction and dynamic response. In this paper, we report on experimental results obtained applying two complex methods in a prototype CMFM, consisting of a commercial flowtube and a SOC based prototype transmitter.

Section 2 briefly reviews the signal processing methods tested in the system. Section 3 introduces the hardware platform. Section 4 describes the single-phase and two-phase flow tests. The two-phase flow results are compared with the performance of an algorithm that is used in a commercial, two-phase capable flow meter.

\section{Complex bandpass filter method}

The signal processing of the CMFM is responsible for filtering noise and tracking the sensor signal's parameters in real time. These

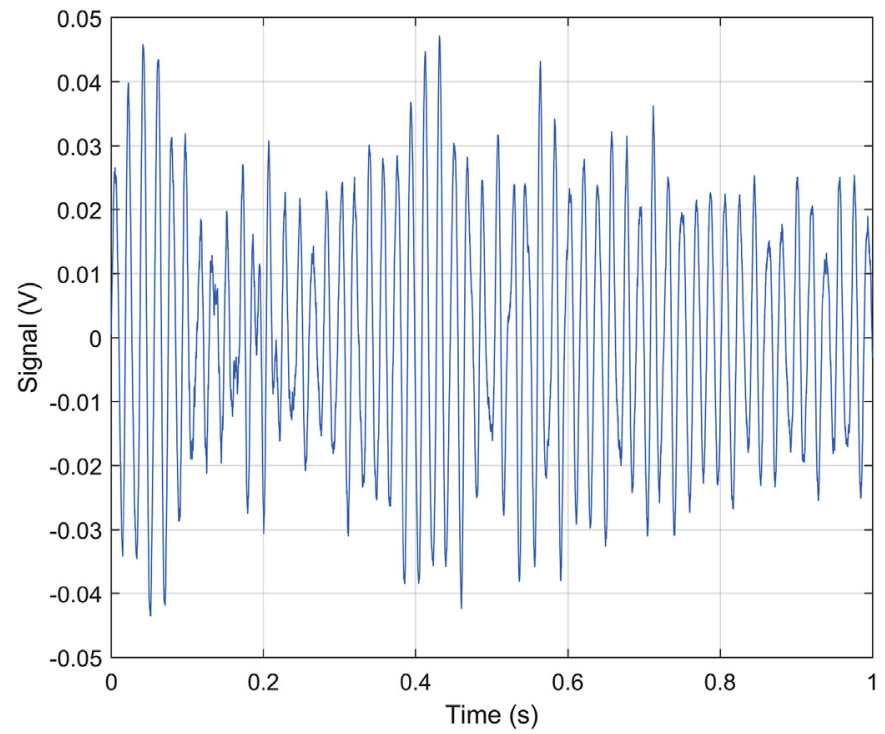

Fig. 3. Sensor signal obtained with $1 \mathrm{~kg} / \mathrm{s}$ water flow rate and $70 \%$ GVF.

parameter values are used for flowtube control and higher level measurement calculations. Fig. 3 shows a typical sensor signal for twophase air/water flow ( $25 \mathrm{~mm}$ flowtube diameter, $1 \mathrm{~kg} / \mathrm{s}$ liquid flow rate with $70 \% \mathrm{GVF}$ ), where the rapid variation in sensor signal amplitude is due to high and varying damping on the flowtube caused by two-phase flow.

The Complex Bandpass Filter (CBF), Complex Notch Filter (CNF) and their combination (CBF-CNF) are introduced in our earlier paper [33]. Here, an overview of their derivation and properties is given to provide context for the experimental work described later in the paper.

In the $\mathrm{CBF}$, a complex shift factor $e^{j \theta}$ is applied to the filter coefficients of an ordinary low-pass filter design (for example based on an elliptic filter). This procedure has two effects. Firstly, the shift in frequency converts the low pass into a bandpass filter, which can be designed to match the operating frequency range of the Coriolis meter. Secondly, the real and imaginary outputs of the filter form an analytic signal (i.e. consisting of an orthogonal sine/cosine pair), so that the sinusoidal properties of the outputs, such as frequency, phase and amplitude, are readily derived on a sample-by-sample basis. By tracking both sensor signals simultaneously, the phase difference (and hence mass flow rate) is readily calculated from the phases of the individual signals.

One way of viewing the construction of the $\mathrm{CBF}$ is that the frequency shift results in the suppression of the negative frequency component which results in the analytic form. In the CNF approach, the negative frequency component is removed using a notch instead of a bandpass filter. This has the advantage that the tracking delay may be very small at the (positive) resonant frequency of the flow tube. Given that a CMFM typically has a range of resonant frequencies, this idea may be generalised by creating a bandstop filter in the negative frequency domain. The CBF and CNF concepts remain distinct however: in the $\mathrm{CBF}$ a low pass filter is shifted higher so that the negative frequency range is excluded, while in the CNF a high-pass filter (with a stopband around $0 \mathrm{~Hz}$ ) is shifted lower so that the stopband covers the desired negative frequency range.

While the reduced delay of the CNF is advantageous, it offers no filtering over positive frequencies, making the technique vulnerable to noise. Accordingly, a combined approach (CBF-CNF) can be used whereby the CNF stage is used primarily to block the negative frequency range and hence generate the analytic signal, while the CBF element provides the desired noise attenuation. The CBF-CNF approach provides additional flexibility to select a suitable balance between tracking delay and noise reduction. 


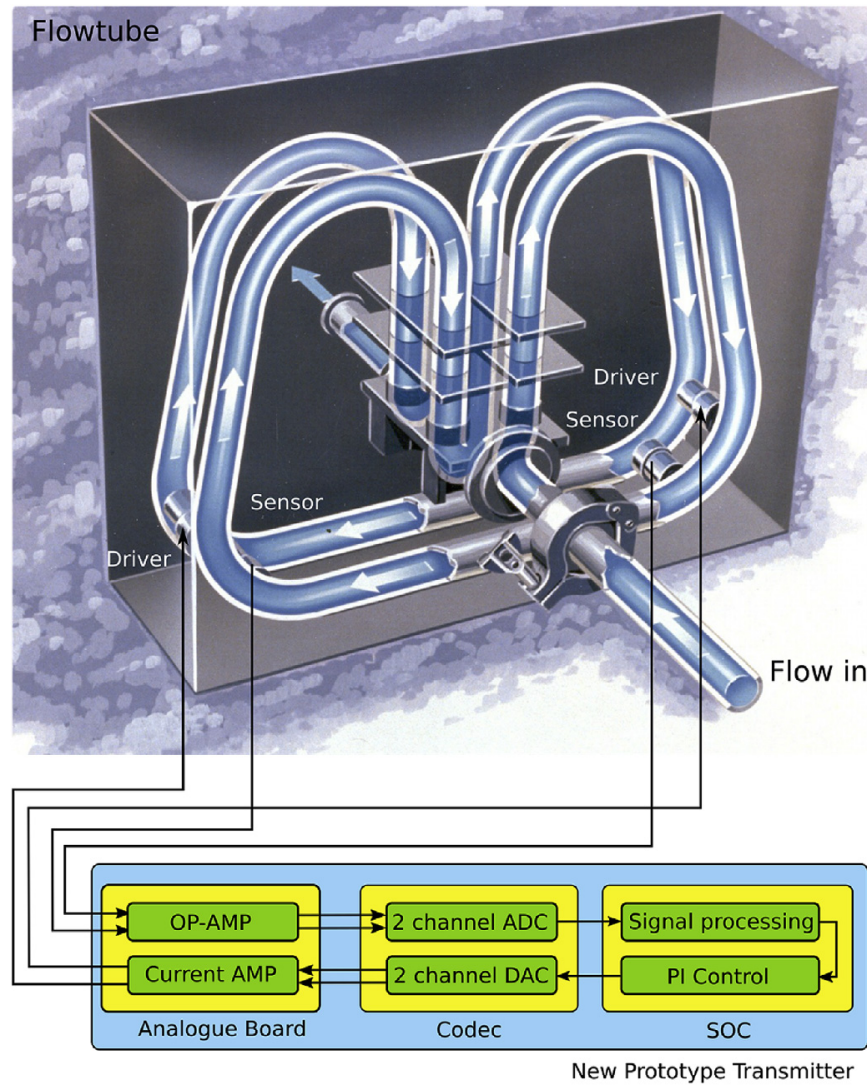

Fig. 4. Diagram of CMFM, including system-on-chip.

In Ref. [33], simulation studies are carried out to compare the performance of the CBF, CNF and CBF-CNF algorithms with well-established techniques for Coriolis signal tracking. A $2 \mathrm{kHz}$ sampling rate was used to match the typical rate used in these prior techniques, and this proved adequately fast to successfully track simulated sensor signals with a nominal resonant frequency of around $100 \mathrm{~Hz}$. In noisy conditions, such as during two-phase flow, the pure CNF technique was shown to perform poorly, and so is not considered further in this paper.

\section{Digital transmitter hardware platform}

The CBF and CBF-CNF algorithms have been implemented to run on a dual ARM core Xilinx Zynq 7000 series FPGA programmed in $\mathrm{C}++$. As shown in Fig. 4, the CMFM consists of two components: a vibrating flow tube in series with the process piping, and an electronic transmitter that maintains flow tube vibration, performs measurement calculations, and transmits measurement data to the user. In the prototype transmitter, we have implemented the complex bandpass filtering signal processing methods alongside a digital drive to maintain flow tube oscillation. The two velocity sensors detect the flow tube motion. Their signals are amplified and sampled by a two-channel Analogue-toDigital Converter (ADC). Processing algorithms are applied to the sensor signals to track their amplitude, frequency and phase. Based on the tracked parameters, a digitally synthesized driver signal is generated by PI control algorithm and Digital-to-Analogue Converter (DAC). Note that the PI amplitude control and drive synthesis techniques used here are broadly similar to those described in Ref. [30]. Finally, the driver signal is amplified by current amplifiers before being applied to the flow tube drive coils. For measurement purposes, the frequency of oscillation is used to calculate the density of the process fluid, while the phase difference between the two sensor signals is used (along with the frequency) to calculate the mass flow rate.

The hardware platform is shown in Fig. 5. The digital board is an

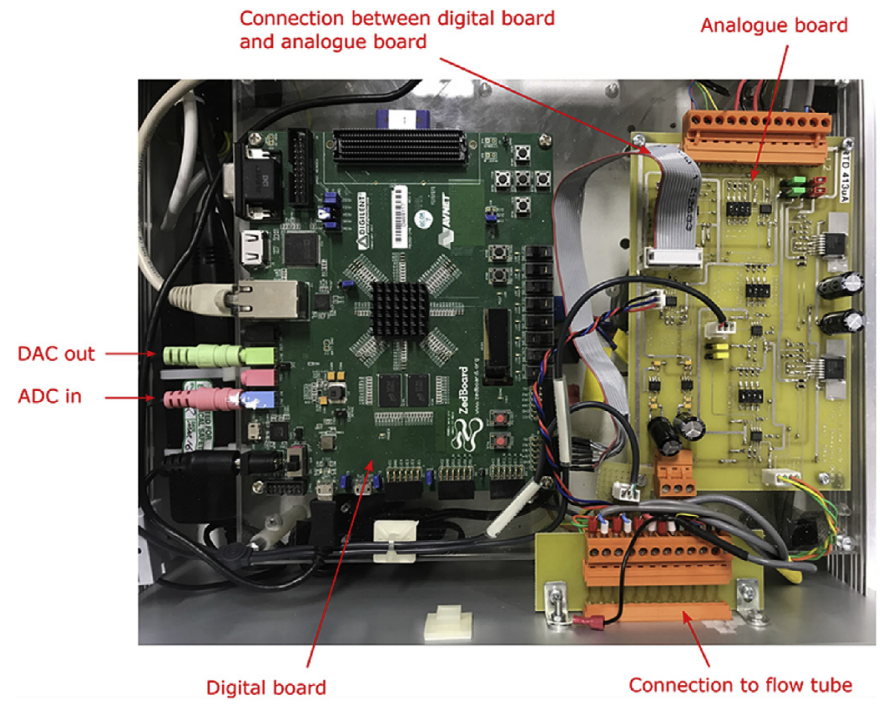

Fig. 5. Hardware platform for transmitter.

off-the-shelf Zynq prototyping card called the Zedboard [37], which provides the FPGA/processor and peripherals including two 24-bit ADCs and two 24-bit DACs with $48 \mathrm{kHz}$ sampling rate. The digital board is responsible for sensor data sampling, measurement calculation, drive signal generation and so on. The analogue board was developed inhouse to provide a suitable interface to Coriolis flow tubes, containing operational amplifiers, analogue peripherals etc.

In the following experimental results, the ADCs and DACs and the tracking algorithms all operate at $48 \mathrm{kHz}$ i.e. the estimates of frequency, amplitude and phase for each of the two sensor signals are updated every sample. This provides a fair basis for comparison with the 'OxBox', an older research transmitter [30], which implements a zerocrossing based signal tracking algorithm which has been used in commercial multiphase Coriolis products (e.g. Ref. [31]). However, although the OxBox processes data at $48 \mathrm{kHz}$, it generates measurement results only at zero crossings i.e. twice every period of the flowtube vibration. This update rate is therefore comparatively slow, and varies with the resonant frequency.

The flowtube used is a Foxboro $25 \mathrm{~mm}$ diameter design. Conventionally, this flowtube is driven in its second mode of vibration (at around $82 \mathrm{~Hz}$ for pure water). In this experimental work the lowest mode of vibration is used - at around $55 \mathrm{~Hz}$ - so the CBF and CNF-CBF filters have been designed accordingly. Further consideration is given to the role of the higher modes of flow tube vibration in the concluding section of the paper.

\section{Experiments}

Initially, single phase experiments were performed to calibrate the prototype and demonstrate good flowmeter performance. Subsequently, two-phase flow experiments were carried out to evaluate the performance of the CBF and CBF-CNF methods against the OxBox.

\subsection{Single-phase experiments}

Firstly, single-phase tests have been carried out against a reference meter in order to verify the linearity between the time delay observed and the true mass flow rate and hence to prove the basic functionality of new prototype transmitter. The single-phase experimental setup is shown in Fig. 6.

In the figure, a commercial CMFM is used as a reference meter. This meter was calibrated at the UK National Flow Laboratory NEL with an uncertainty of approximately $0.1 \%(\mathrm{k}=2)$ over the flow ranges used in these experiments. The pump and reference CMFM are connecting to a 


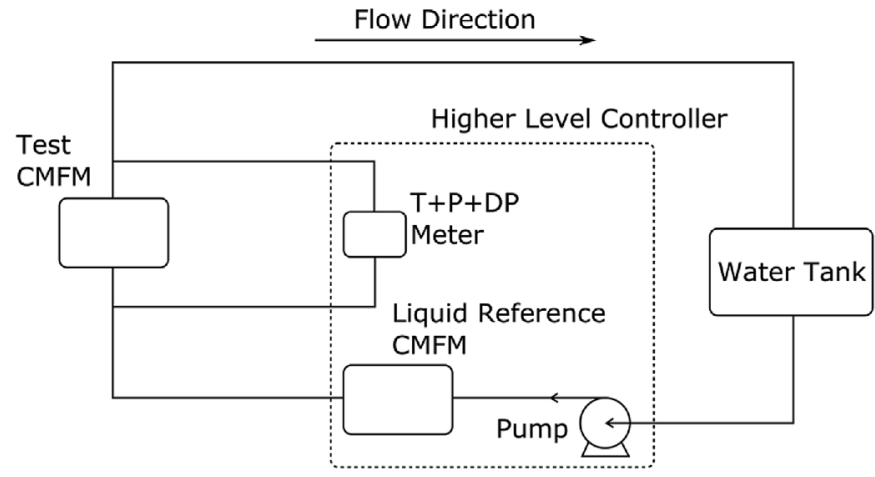

Fig. 6. Single-phase experiment facility.

controller which regulates the experimental conditions. The prototype transmitter is coupled with a $25 \mathrm{~mm}$ diameter commercial flow tube.

Single-phase flow tests were carried out at $0 \mathrm{~kg} / \mathrm{s}, 1 \mathrm{~kg} / \mathrm{s}, 2 \mathrm{~kg} / \mathrm{s}$, $3 \mathrm{~kg} / \mathrm{s}$ and $4 \mathrm{~kg} / \mathrm{s}$. The phase difference and hence time difference calculated by the CBF and CBF-CNF algorithms were recorded in each experiment. A linear fit is used to verify the linearity between the reference flow rate and the measured time difference. Note that other factors - such as fluid density, pressure and temperature - which may affect the time difference/flow rate relationship, are assumed constant in these tests. The results are shown in Fig. 7.

From the figure, the time difference generated by both the CBF and CBF-CNF algorithms track the reference flow rate well; assuming a linear relationship the residual errors remain within approximately \pm 1e-7s. If temperature and pressure are taken into account, this error is expected to be reduced further. These results demonstrate that the prototype transmitter, following the basic physical principle of the CMFM, generate acceptable measurement results for single-phase flow.

\subsection{Two-phase experiments}

After validating the newly developed Coriolis flowmeter prototype for single-phase flow, two-phase flow (water mixed with air) tests have been carried out using different liquid flow rates and different Gas Void Fractions (GVF). The two-phase flow experiment facility diagram is shown in Figs. 8 and 9. Compressed air is metered and mixed with water to create a two-phase flow. Reference CMFMs together with a pump (water) and valve (air) are connected to a higher level controller. The inlet pressure and the differential pressure across the flowtube are
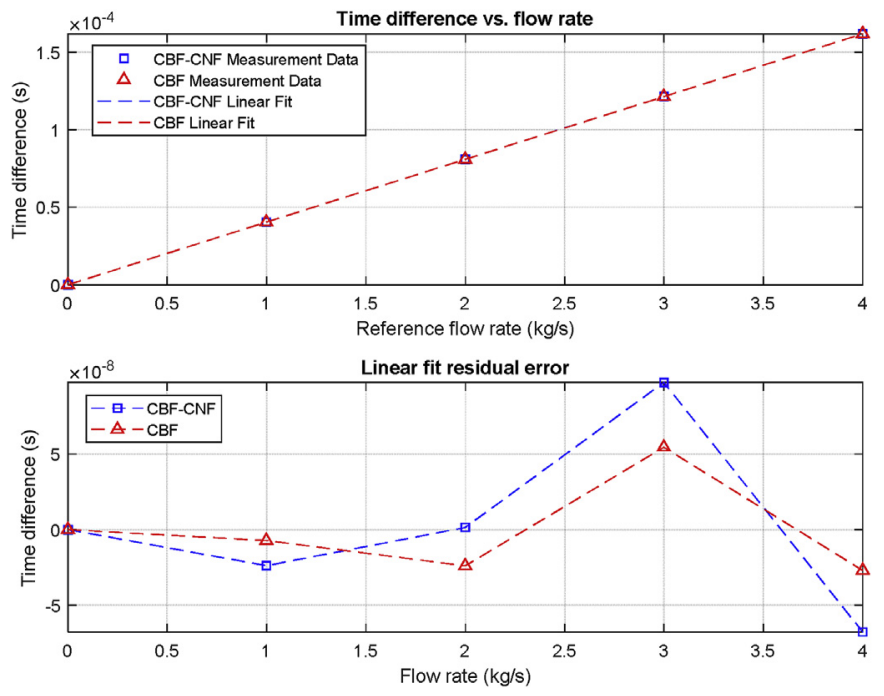

Fig. 7. Linear fitting for measured phase difference and mass flow rate.

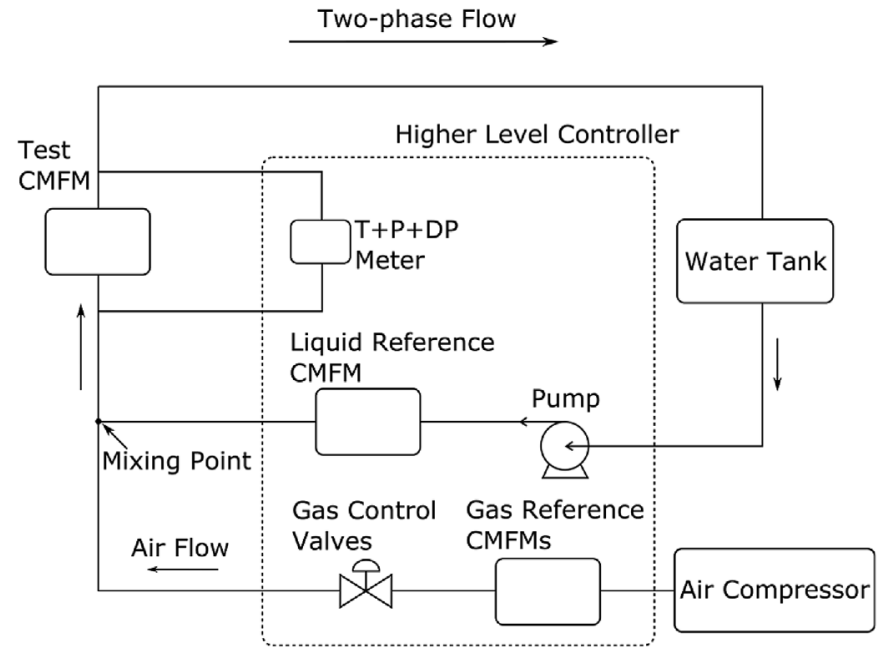

Fig. 8. Two-phase flow experiment facility.

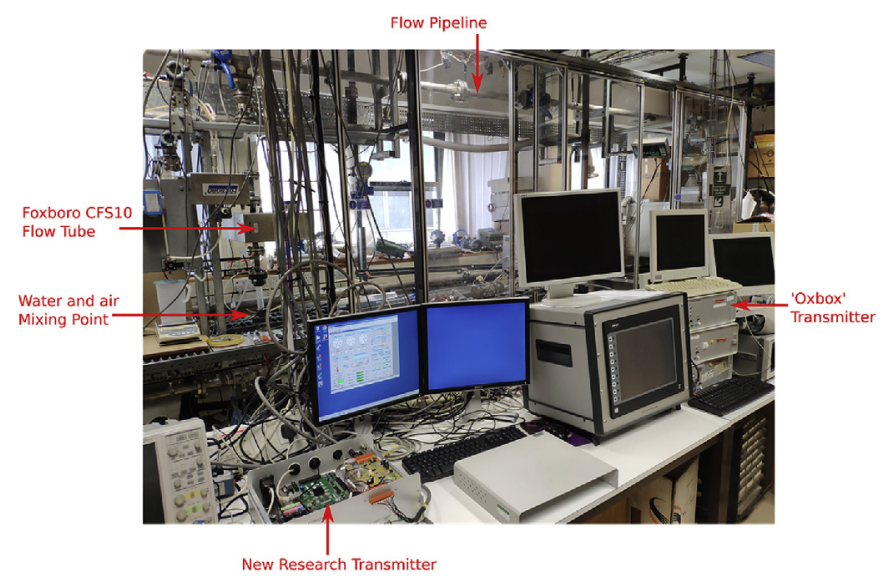

Fig. 9. Experiment setup for single-phase and two-phase flow tests.

measured by the $\mathrm{T}+\mathrm{P}+\mathrm{DP}$ (Temperature, Pressure, Differential Pressure) sensor so that the local GVF, which is a function of gas volume and therefore of the water/air mixture pressure, can be calculated. As the injection of air results in an increase in back-pressure on the water flow, independent control loops are used to maintain on the one hand the desired liquid flow rate (by adjusting the variable speed drive on the water pump) and on the other hand the GVF (by adjusting the mass flow rate of the gas via a set of Coriolis based gas flow controllers, where the desired gas mass flow is calculated from the desired GVF and the current pressure at the flowtube entrance). In these experiments the pressure at the flowtube is not controlled, and, for a fixed flow rate, it exhibits a gradual rise as the GVF increases. However, this inlet pressure can also be kept steady if required by including a further independent control loop, for example by controlling a valve downstream of the flowtube.

There is a complex relationship between the pattern of two-phase flow passing through the flowtube and the resulting sensor signals (for example as illustrated in Fig. 3). The most important source of amplitude variation is the damping along the flowtube, which for two-phase flow is likely to be non-uniform [15], rapidly changing, and typically one or more orders of magnitude higher than for single phase flow. With many flowtube designs including a flow splitter at the inlet, the pattern of flow through the complex geometry may not even be repeatable. To maintain flowtube oscillation, the control system provides a drive signal, which introduces additional dynamics. These strong amplitude dynamics in turn influence the instantaneous frequency and phase of the flowtube oscillation. Overall, therefore, other than for 


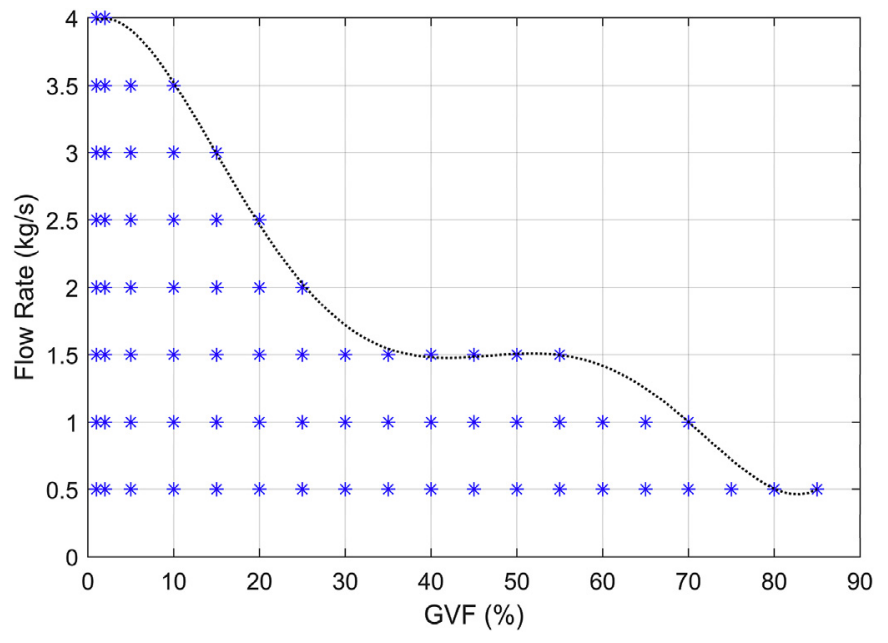

Fig. 10. Two-phase flow experiment testing points. The maximum GVF at each flow rate is determined by limiting the pressure drop across the $25 \mathrm{~mm}$ Foxboro CFS-10 flow tube to below $150 \mathrm{kPa}$.

certain slug flow conditions where the passage of liquid or gas slugs are observable in the sensor signals, correlation between flow distribution and sensor signal behaviour is not established, and as discussed in Ref. [33], changes in amplitude frequency and phase are currently best modelled simply as filtered white noise.

The pressure drop depends on tube design, i.e. primarily the length of the tube and its inner diameter [38], as well as the two-phase flow conditions. A combination of high flow and high GVF can lead to an impractically large pressure drop across the flow-tube [24]. For testing two-phase performance, water flow rates from $0.5 \mathrm{~kg} / \mathrm{s}$ up to $4 \mathrm{~kg} / \mathrm{s}$ are used in steps of $0.5 \mathrm{~kg} / \mathrm{s}$ while the GVF varies from $1 \%$ up to $85 \%$. At each flow rate, the maximum GVF was selected to restrict the pressure drop below $150 \mathrm{kPa}$, for a $25 \mathrm{~mm}$ Foxboro CFS-10 flowtube. The resulting test grid for two-phase flow experiments and the nominal pressure drop limit line are shown in Fig. 10.

When assessing the performance of frequency, amplitude and phase difference tracking in two-phase flow experiments, and their subsequent influence on the mass flow and density measurements, a number of difficulties arise. The first is the absence of any 'true' reference measurements: given the random nature of two-phase flow, the instantaneous values of the sensor signal parameters are not known. However, our previous study has evaluated the algorithms' performance with simulated two-phase data, where the true, time-varying signal parameter values are known [33]. The second difficulty is that, with real flow experiments, even though reference measurements are available for the time-averaged mass flow and density, 'true' instantaneous values (i.e. the exact mixture passing through the flowtube at the current time) are also not available. A third difficulty is that, in addition to the short-term random variations, it is well established that there are systematic errors in the average mass flow and density measurements with two-phase flow, requiring modelling and correction [23]. Finally, the frequency, amplitude and phase measurements are complicated by the closed loop control of the flow tube oscillation - their variations are a function of the control loop responsiveness as well as the time delay and errors associated with the measurement algorithms.

Given these difficulties, the approach taken to assess the performance of the CBF and CBF-CNF algorithms is as follows: for a given set of experimental conditions (liquid flow rate and GVF), the standard deviation of frequency, phase difference and amplitude are compared with those generated by the Oxbox, working with the same flow tube in the same experimental conditions. It is assumed that, given the same flow tube and experimental conditions, and a broadly similar flow tube control strategy (programmed by the same team in each case), any improvement in measurement quality (as indicated by reduced

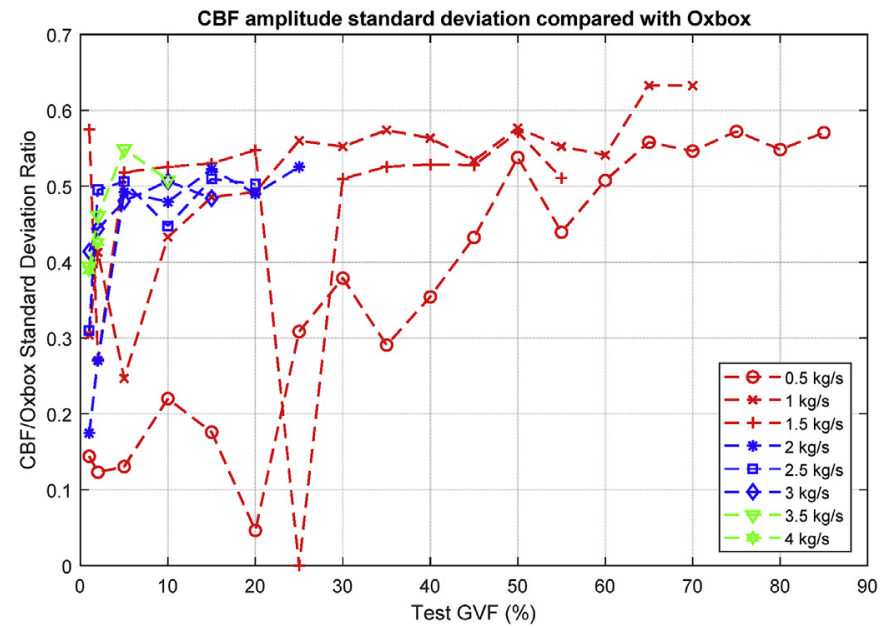

Fig. 11. Amplitude standard deviation comparison between CBF and Oxbox.

standard deviation) will be attributable to an improvement in the signal tracking algorithms, in terms of accuracy and/or reduced delay, rather than any other factor.

To assess the performance of the complex signal processing methods, we calculate the ratio between the CBF and CBF-CNF standard deviations and those of the Oxbox transmitter for amplitude, frequency and phase difference. Thus a value below 1.0 indicates that the standard deviation for the complex signal processing technique is lower than that generated by the Oxbox. This approach needs careful interpretation: for example, the application of a simple low pass filter can potentially reduce the observed standard deviation of a time series, but only at the cost of a reduced dynamic response (and hence tracking ability). Here, as illustrated in simulation studies [33], the complex methods have a faster dynamic response than the zero-crossing technique; furthermore, the standard deviations are based upon a $48 \mathrm{kHz}$ update rate (as opposed to approximately $200 \mathrm{~Hz}$ for the zero crossing technique - the exact update rate varies with the resonant frequency). Accordingly, it is reasonable to claim that a ratio below 1.0 indicates an improved measurement performance.

The measurement results for CBF for all test points are shown in Figs. $11-13$. Note that at $1.5 \mathrm{~kg} / \mathrm{s}$ water flow rate and $25 \% \mathrm{GVF}$, the Oxbox cannot maintain flow tube vibration and the ratio is given as zero. The amplitude standard deviation (Fig. 11) is consistently reduced against the Oxbox. This demonstrates a combination of better measurement resulting in better flow tube control using the new signal processing techniques. The frequency results (Fig. 12) are similar to

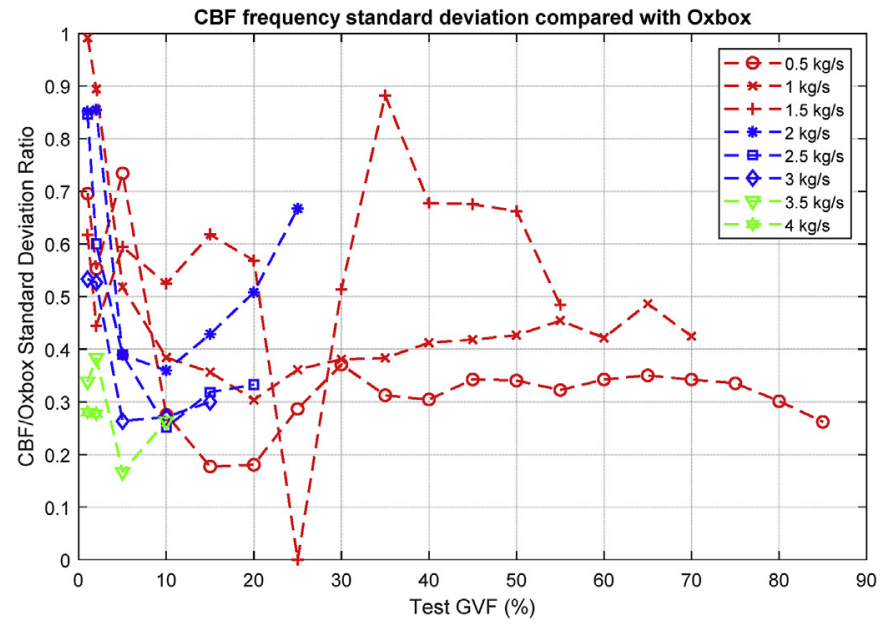

Fig. 12. Frequency standard deviation comparison between CBF and Oxbox. 


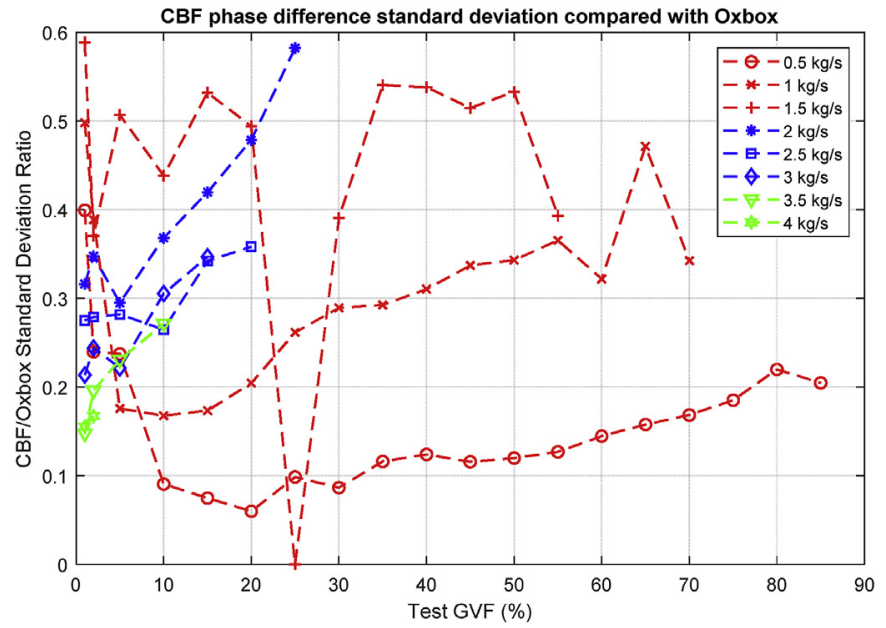

Fig. 13. Phase difference standard deviation comparison between CBF and Oxbox.

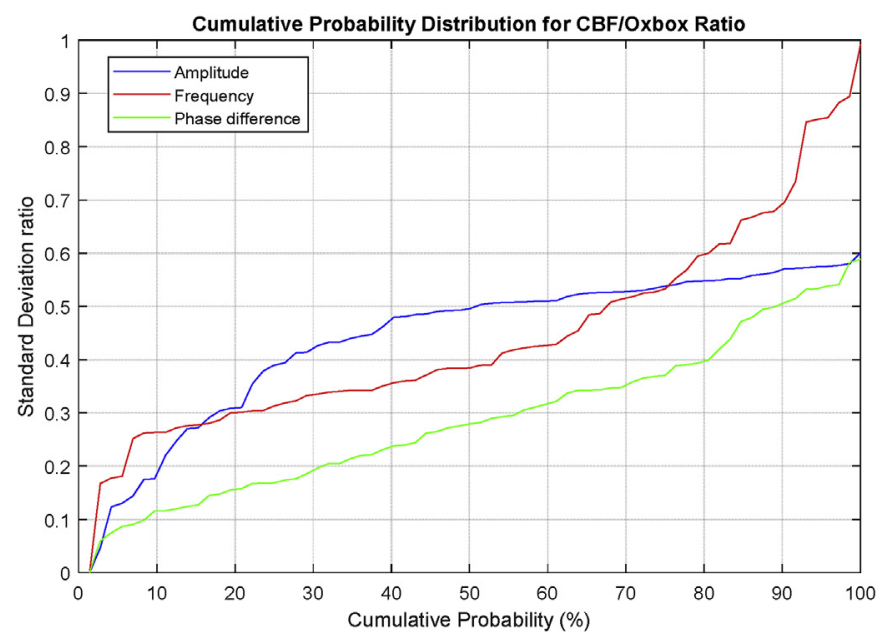

Fig. 14. Cumulative probability distribution of CBF/Oxbox ratios.

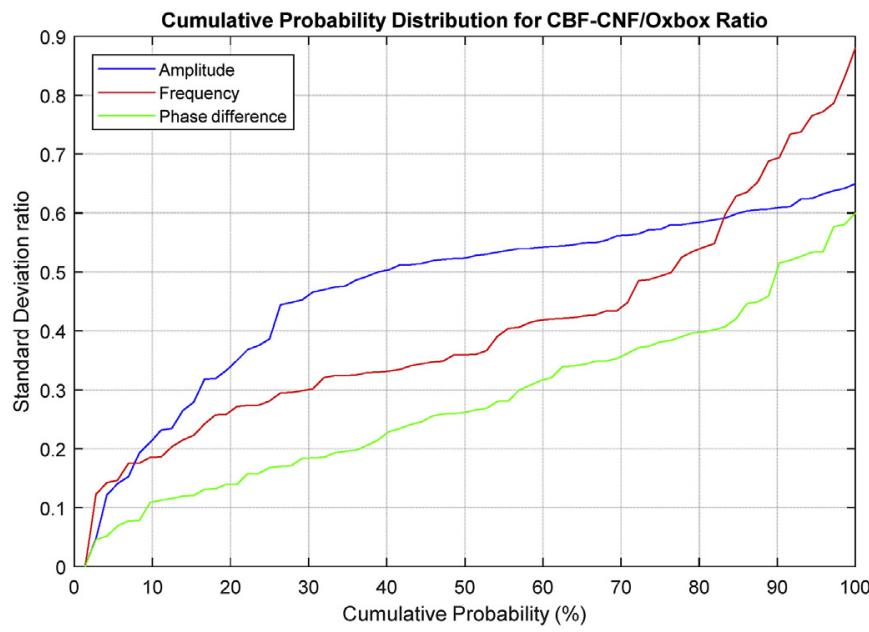

Fig. 15. Cumulative probability distribution of CBF-CNF/Oxbox ratios.

those for amplitude. The phase difference results (Fig. 13) show up to 10 times improvement over the Oxbox for low flow and moderate levels of GVF.

Over the eight flow lines a wide variety of standard deviation ratios are generated. In order to summarise the performance across different

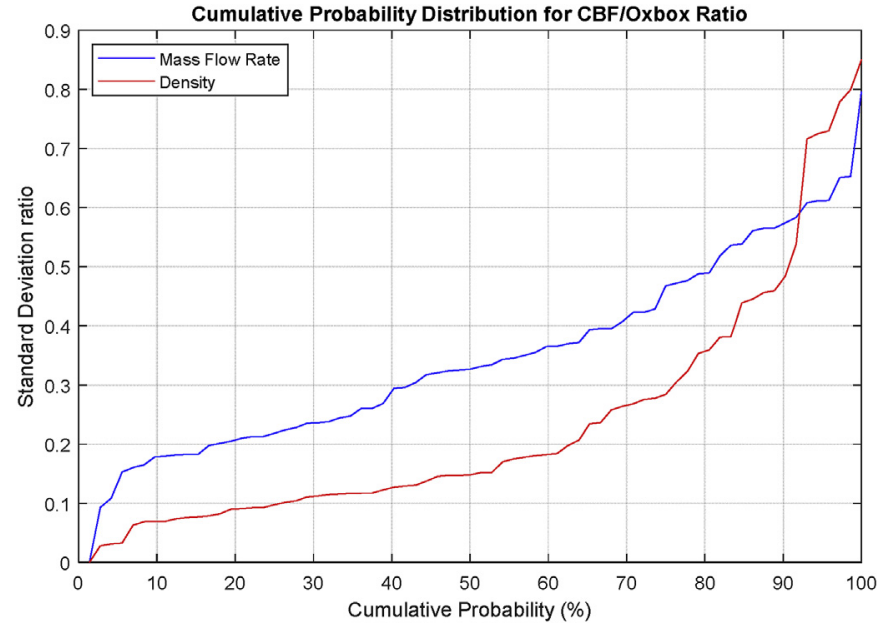

Fig. 16. Result of mass flow rate and density standard deviation ratio.

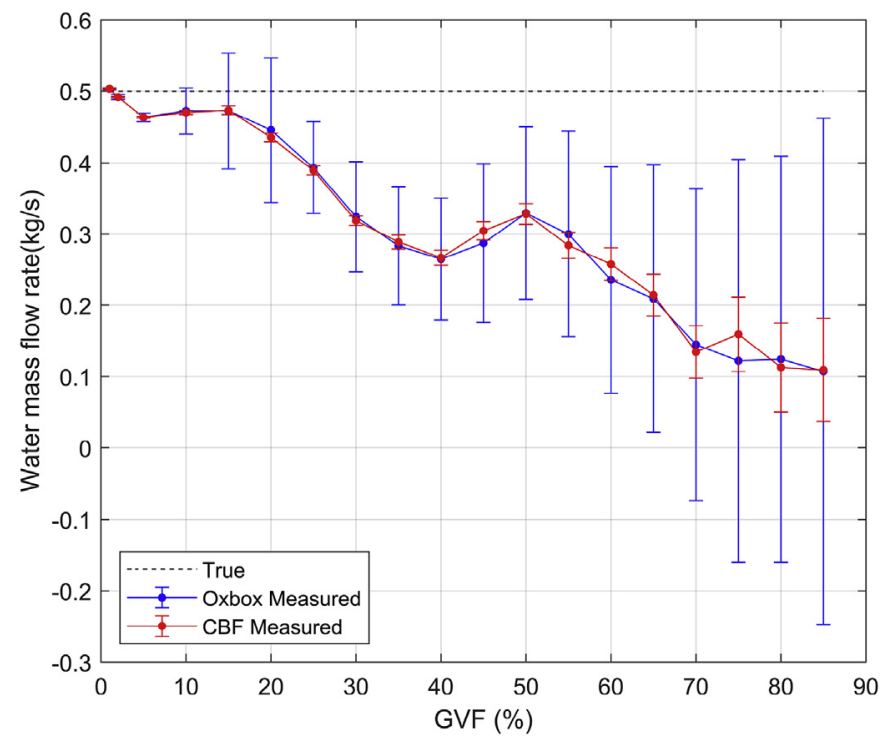

Fig. 17. Comparison of standard deviations for Oxbox and CBF techniques for mass flow rate at $0.5 \mathrm{~kg} / \mathrm{s}$.

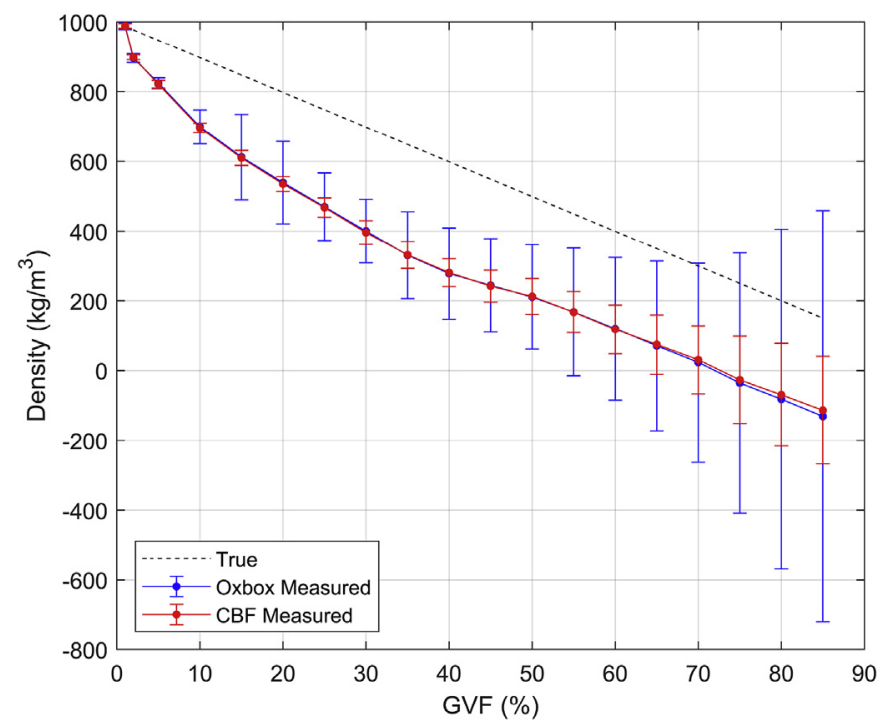

Fig. 18. Comparison of standard deviations for Oxbox and CBF techniques for density at $0.5 \mathrm{~kg} / \mathrm{s}$. 
liquid mass flow rates and GVFs, the cumulative probability distribution for each parameters' standard deviation ratio for both CBF and CBF-CNF have been calculated, as shown in Figs. 14 and 15. Each line shows, for the corresponding parameter and complex calculation method, what percentage of experimental points (y-axis) have a standard deviation ratio less than the $\mathrm{x}$-axis value. From the figures, the amplitude standard deviation for both CBF and CBF-CNF are roughly 2 times better than the Oxbox in $50 \%$ of the experiments. For frequency, the $\mathrm{CBF}$ and $\mathrm{CBF}-\mathrm{CNF}$ methods are roughly 2.5 times better than the Oxbox for $50 \%$ of the experiments. For phase difference, $50 \%$ of the experiments show at least 3 times improvement.

Figs. 14 and 15 show the CBF and CBF-CNF performance are broadly similar, with some systematic improvement of CBF over CBFCNF for amplitude, possibly due to its deeper stopband attenuation. This suggests that to achieve improved flowtube control during twophase flow, both the dynamic response and the noise suppression capabilities of measurement algorithms are important.

Finally, the results for the signal tracking are propagated through into the flow and density measurements to see the resulting reductions in standard deviation as shown in Fig. 16.

For the mass flow rate result, the improvement is similar to that of phase difference, where $80 \%$ values are 2 times better than the Oxbox, and all results show at least a 1.2 times improvement. Furthermore, $50 \%$ of results are at least 3 times better than Oxbox. For density, the improvement is very promising, with $50 \%$ of results at least 6 times better than the Oxbox, and $90 \%$ of the results showing at least a 2 times improvement.

To illustrate how the improved results are reflected in reduced fluid measurement errors, Figs. 17 and 18 repeat the results from Figs. 1 and 2 with new CBF results superimposed. They show the mean and standard deviations for mass flow rate and density obtained from the OxBox (blue) and CBF (red) for the $0.5 \mathrm{~kg} / \mathrm{s}$ water flow experiments. The reduction in standard deviation delivered by the CBF is clearly indicated.

Overall, these results demonstrate that $\mathrm{CBF}$ and CBF-CNF methods can operate successfully in real time over a range of liquid mass flow rates and GVFs. Furthermore, significant improvements are achieved for $\mathrm{CBF}$ and CBF-CNF methods over the Oxbox zero-crossing technique, which has previously been used in a wide range of two-phase applications.

\section{Conclusion}

In this paper, we have implemented a family of complex signal processing techniques on a prototype CFMF transmitter. From singlephase flow tests, we have demonstrated that the CBF and CBF-CNF algorithms provide acceptable measurement performance when running on the prototype transmitter connected to a commercial flow tube. The two-phase flow test results demonstrate that the new algorithms deliver reduced standard deviations compared with an established algorithm that has been used in two-phase and multiphase applications.

One issue not directly addressed in this approach is the presence of additional vibrational modes in the flow tube, which are readily excited in the presence of two-phase flow [39,40], or other mechanical noise [41], and are likely to be a more important noise source then general broadband noise. If the nearest mode to the drive frequency is sufficiently distant, then this is relatively easily dealt with using the bandpass mechanism described here. However, if the nearest mode is relatively close and/or if this mode is below the drive frequency (as is the case for the conventional operation of the Foxboro flowtube), then additional filtering stages may be required, for example additional notch filtering at the positive frequency range associated with the undesired mode of vibration. This issue will be further explored in future work.

\section{Acknowledgement}

The Chinese Scholarship Council (Grant id: 201508060350).

\section{References}

[1] K. Adefila, Y. Yan, L. Sun, T. Wang, Calibration of an averaging pitot tube for gaseous CO2 flowmetering, IEEE Trans. Instrum. Meas. 64 (5) (May 2015) 1240-1249.

[2] M. Kazahaya, A mathematical model and error analysis of coriolis mass flowmeters, IEEE Trans. Instrum. Meas. 60 (4) (Apr. 2011) 1163-1174.

[3] S. Panigrahy, N.K. Mishra, S.C. Mishra, P. Muthukumar, Numerical and experimental analyses of LPG (liquefied petroleum gas) combustion in a domestic cooking stove with a porous radiant burner, Energy 95 (Jan. 2016) 404-414.

[4] L. Wang, J. Liu, Y. Yan, X. Wang, T. Wang, Gas-liquid two-phase flow measurement using coriolis flowmeters incorporating artificial neural network, support vector machine, and genetic programming algorithms, IEEE Trans. Instrum. Meas. 66 (5) (May 2017) 852-868.

[5] F. Leach, S. Karout, F. Zhou, M. Tombs, M. Davy, M. Henry, Fast Coriolis mass flow metering for monitoring diesel fuel injection, Flow Meas. Instrum. 58 (Dec. 2017) $1-5$.

[6] X. Lei, W. Zhang, J. Zhang, N. Dinh, H. Li, Experimental investigations on the boiling heat transfer of horizontal flow in the near-critical region, Int. J. Heat Mass Transf. 125 (Oct. 2018) 618-628.

[7] G. Verma, R.K. Prasad, R.A. Agarwal, S. Jain, A.K. Agarwal, Experimental investigations of combustion, performance and emission characteristics of a hydrogen enriched natural gas fuelled prototype spark ignition engine, Fuel 178 (Aug. 2016) 209-217.

[8] M.V.P. Carneiro, P.A. de Oliveira, J.R. Barbosa, A compact refrigeration system based on multijet sprays for electronics thermal management, Exp. Therm. Fluid Sci. 97 (Oct. 2018) 180-191.

[9] G. Yang, H. Hu, G. Ding, J. Chen, W. Yang, S. Hu, Influence of component proportion on heat transfer characteristics of ethane/propane mixture flow condensation in shell side of helically baffled shell-and-tube heat exchanger, Exp Therm. Fluid Sci. 97 (Oct. 2018) 381-391.

[10] R.J. Reizner, Coriolis - the almost perfect flow meter, Comput. Contr. Eng. J. 14 (4) (Sep. 2003) 28-33.

[11] M.P. Henry, et al., A self-validating digital Coriolis mass-flow meter: an overview, Contr. Eng. Pract. 8 (5) (2000).

[12] Q.-L. Hou, K.-J. Xu, M. Fang, C. Liu, W.-J. Xiong, Development of Coriolis mass flowmeter with digital drive and signal processing technology, ISA Trans. 52 (5) (Sep. 2013) 692-700.

[13] L. Wang, Y. Yan, X. Wang, T. Wang, Q. Duan, W. Zhang, Mass flow measurement of gas-liquid two-phase $\mathrm{CO} 2$ in CCS transportation pipelines using Coriolis flowmeters, Int. J. Greenh. Gas Control 68 (Jan. 2018) 269-275.

[14] J.A. Weinstein, The Motion of Bubbles and Particles in Oscillating Liquids with Applications to Multiphase Flow in Coriolis Meters, PhD Thesis University of Colorado, 2008.

[15] N.T. Basse, A review of the theory of Coriolis flowmeter measurement errors due to entrained particles, Flow Meas. Instrum. 37 (Jun. 2014) 107-118.

[16] N.T. Basse, Coriolis flowmeter damping for two-phase flow due to decoupling, Flow Meas. Instrum. 52 (Dec. 2016) 40-52.

[17] J. Liu, T. Wang, Y. Yan, X. Wang, L. Wang, Investigations into the behaviours of Coriolis flowmeters under air-water two-phase flow conditions on an optimized experimental platform, 2018 IEEE Int. Instrum. Meas. Technol. Conf. 2018, pp. 1-6.

[18] J. Hemp, J. Kutin, Theory of errors in Coriolis flowmeter readings due to compressibility of the fluid being metered, Flow Meas. Instrum. 17 (6) (Dec. 2006) 359-369.

[19] D.L. Gysling, An aeroelastic model of Coriolis mass and density meters operating on aerated mixtures, Flow Meas. Instrum. 18 (2) (Apr. 2007) 69-77.

[20] J. Hemp, G. Sultan, On the theory and performance of Coriolis mass flowmeters, Int. Conf. Mass Flow Meas. Direct Indirect. Coriolis metering I. London, Proceedings of the International Conference on Mass Flow Measurement, 1989, pp. 21-22.

[21] J.A. Weinstein, D.R. Kassoy, M.J. Bell, Experimental study of oscillatory motion of particles and bubbles with applications to Coriolis flow meters, Phys. Fluids 20 (10) (Oct. 2008) 103306.

[22] L. Xing, Y. Geng, C. Hua, H. Zhu, A. Rieder, W. Drahm, M. Bezdek, A combination method for metering gas-liquid two-phase flows of low liquid loading applying ultrasonic and Coriolis flowmeters, Flow Meas. Instrum. 37 (Jun. 2014) 135-143.

[23] R.P. Liu, M.J. Fuent, M.P. Henry, M.D. Duta, A neural network to correct mass flow errors caused by two-phase flow in a digital coriolis mass flowmeter, Flow Meas. Instrum. 12 (1) (Mar. 2001) 53-63.

[24] M. Henry, M. Tombs, M. Duta, F. Zhou, R. Mercado, F. Kenyery, J. Shen, M. Morles, C. Garcia, R. Langansan, Two-phase flow metering of heavy oil using a Coriolis mass flow meter: a case study, Flow Meas. Instrum. 17 (6) (Dec. 2006) 399-413.

[25] R. Thorn, G.A. Johansen, B.T. Hjertaker, Three-phase flow measurement in the petroleum industry, Meas. Sci. Technol. 24 (1) (Jan. 2013) 012003.

[26] Q.-L. Hou, K.-J. Xu, M. Fang, Y. Shi, B.-B. Tao, R.-W. Jiang, Gas-Liquid two-phase flow correction method for digital CMF, IEEE Trans. Instrum. Meas. 63 (10) (Oct. 2014) 2396-2404.

[27] L. Wang, Y. Yan, X. Wang, T. Wang, Input variable selection for data-driven models of Coriolis flowmeters for two-phase flow measurement, Meas. Sci. Technol. 28 (3) (Mar. 2017) 035305.

[28] L. Wang, J. Liu, Y. Yan, X. Wang, T. Wang, Gas-liquid two-phase flow measurement 
using coriolis flowmeters incorporating artificial neural network, support vector machine, and genetic programming algorithms, IEEE Trans. Instrum. Meas. 66 (5) (May 2017) 852-868.

[29] G. R. Duffill, S. M. Jones, and A. T. Patten, "Meter Electronics and Methods for Determining a Liquid Flow Fraction in a Gas Flow Material,” U.S. Patent 7974792, July $05,2011$.

[30] M. Zamora, M.P. Henry, An FPGA implementation of a digital coriolis mass flow metering drive system, IEEE Trans. Ind. Electron. 55 (7) (Jul. 2008) 2820-2831.

[31] M. Henry, M. Tombs, F. Zhou, Field experience of well testing using multiphase Coriolis metering, Flow Meas. Instrum. 52 (Dec. 2016) 121-136.

[32] M. Li, M. Henry, Complex bandpass filtering for Coriolis mass flow meter signal processing, IECON 2016 - 42nd Annu. Conf. IEEE Ind. Electron. Soc. 2016, pp. 4952-4957.

[33] M. Li, M. Henry, Complex signal processing for Coriolis mass flow metering in twophase flow, Flow Meas. Instrum. 64 (Dec. 2018) 104-115.

[34] C. Clark, R. Cheesewright, Experimental determination of the dynamic response of Coriolis mass flow meters, Flow Meas. Instrum. 17 (1) (Mar. 2006) 39-47.

[35] C. Clark, R. Cheesewright, S. Wang, Prediction of the dynamic performance of fast response coriolis meter systems, IEEE Trans. Instrum. Meas. 57 (1) (Jan. 2008)
95-99.

[36] C. Clark, M. Zamora, M. Henry, R. Cheesewright, M. Henry, The dynamic performance of a new ultra-fast response Coriolis flow meter, Flow Meas. Instrum. 17 (6) (Dec. 2006) 391-398.

[37] Zedboard, [Online]. Available: http://www.zedboard.org/ [Accessed: 03-Apr2018].

[38] M. Anklin, W. Drahm, A. Rieder, Coriolis mass flowmeters: overview of the current state of the art and latest research, Flow Meas. Instrum. 17 (6) (Dec. 2006) 317-323.

[39] M.P. Henry, O. Ibryaeva, A. Semenov, Measurement validation for ICPS: matrix pencil method for coriolis metering with liquid/gas flow, 1st IEEE International Conference on Industrial Cyber-Physical Systems (ICPS-2018), 15 May 2018.

[40] M.P. Henry, O. Ibryaeva, P. Taranenko, M. Tombs, F.B. Zhou, Matrix pencil method for coriolis metering with liquid/gas flow II: experimental results, 1st IEEE International Conference on Industrial Cyber-Physical Systems (ICPS-2018), 15 May 2018.

[41] F. Leach, S. Karout, F.B. Zhou, M.S. Tombs, M. Davy, M.P. Henry, Fast Coriolis mass flow metering for monitoring diesel fuel injection, Flow Meas. Instrum. 58 (2017) 1-5. 